\title{
Multi-Modal Communications in Underwater Sensor Networks Using Depth Adjustment
}

\author{
Michael O'Rourke ${ }^{\dagger}$, Elizabeth Basha ${ }^{\dagger}$, and Carrick Detweiler* \\ $\dagger$ University of the Pacific, Stockton, California \\ *University of Nebraska-Lincoln, Lincoln, Nebraska \\ m_orourke@u.pacific.edu,ebasha@pacific.edu,carrick@cse.unl.edu
}

\begin{abstract}
Acoustic communication typically dominates the power usage in underwater sensor networks. As networks underwater have very limited recharging capabilities, this challenges the network's ability to communicate collected data. To balance these conflicting needs, we utilize a sensor network platform with underwater acoustic communication, surface level radio communication, and a depth adjustment system to switch between them. Nodes determine if they should surface to communicate by approximating the network energy usage and data latency given the data transmission size. For a given path, we develop and examine a set of algorithms to select the nodes to rise to communicate the data via radio across the network while taking energy usage into account. We perform a preliminary analysis of the methods and show that for typical networks greedy approaches are nearly as good as centralized approaches, yet require minimal communication overhead and only local information.
\end{abstract}

\section{INTRODUCTION}

Underwater sensor networks face energy challenges in ensuring continued performance and the key drain on energy is communication. However the need to transmit collected data operates in direct conflict with this challenge. Others have utilized data muling or avoided communication by simply storing data. In this paper, we propose a multi-modal communication approach that leverages a novel depth adjustment system, developed in our prior work [7], that allows the sensor nodes to surface to send data using radio.

Our approach allows the sensor nodes to utilize two different communication methods: acoustic for in-water control and radio for data transfer at the surface. Nodes compute the cost trade-off based on the amount data they need to send and the cost of surfacing. When a node determines that it has sufficient data for surface communication, it uses the acoustic modems to tell nodes along the path to surface

Permission to make digital or hard copies of all or part of this work for personal or classroom use is granted without fee provided that copies are not made or distributed for profit or commercial advantage and that copies bear this notice and the full citation on the first page. To copy otherwise, to republish, to post on servers or to redistribute to lists, requires prior specific permission and/or a fee.

WUWNet'12, November 5-6, 2012 Los Angeles, California, USA

Copyright 2012 ACM 978-1-4503-1773-3-3/12/11 ... \$15.00.

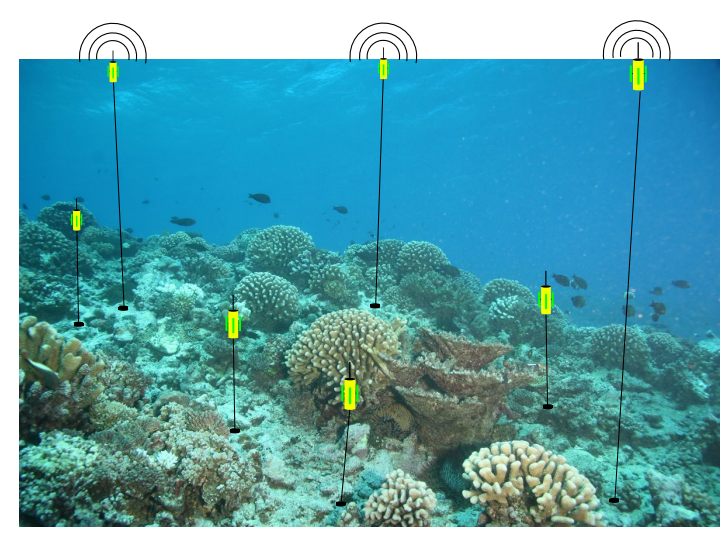

Figure 1: Example where only a subset of nodes must surface to create a radio link between nodes on the left and right.

to create a radio link as shown in Figure 1. The surface route will differ from the acoustic route due to differing communication ranges, so the nodes along the route must first determine which nodes should surface. These nodes surface together, communicate the data, and then descend back to optimal sensing locations.

In this paper, we focus on determining the best subset of nodes along the acoustic route that should surface to participate in the radio communication. We examine the tradeoff between local greedy algorithms and their centralized, optimal counterparts for minimizing energy usage and minimizing hop count. We find that while there are certainly configurations that cause the greedy algorithms to perform poorly, for typical networks the greedy approaches work well, require minimal overhead, and utilize only local information. This motivates our future work where we are examining more realistic cases with many sources and sinks of data as well as overlapping communication routes. In these, the optimal radio routes will likely contain nodes not on the acoustic path; still we hope to show that similar greedy approaches also perform well in these systems.

This paper has the following format. Section 2 describes related literature, Section 3 outlines the AquaNode platform and its communication modalities, and Section 4 analyzes the communication systems. Section 5 then introduces our algorithms for link selection, which Section 6 validates through simulation. Section 7 concludes the paper. 


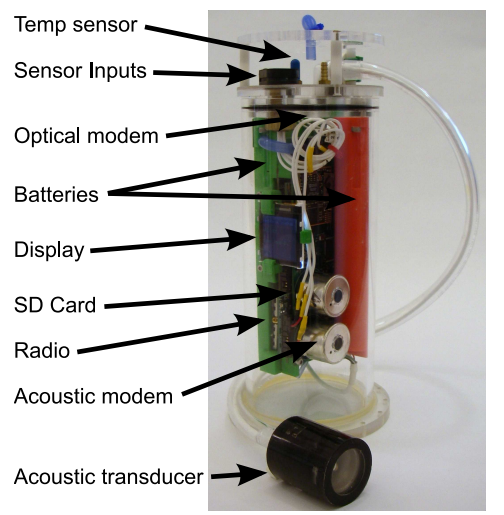

Figure 2: AQUANODE sensor node platform overview.

\section{RELATED WORK}

Many underwater sensor networks leverage surface nodes for long-range, high-throughput communication channels [2]. For instance, the US Navy deployed a system called SeaWeb [9] to test deployments of multi-node underwater networks with static and mobile nodes. SeaWeb had a number of radio/acoustic ("Racom") nodes at the surface that could communicate both acoustically and with radio to satellites, ships, or shore. These Racom nodes also had the advantage of anchoring localization systems since they have access to GPS. Using a surface gateway node is one of the more practical methods to obtain information from an underwater network [5]. Where to place surface gateway nodes to minimize energy and end-to-end delay given a set of underwater nodes with known positions has been examined using integer linear programming [12]. With these types of systems, the overall bandwidth of the system is limited by the acoustic channel, since all nodes need to transmit acoustically to a radio gateway node. Another option for obtaining data from an underwater sensor network is to use an underwater vehicle to collect the data. Underwater gliders surface periodically and send data collected along their trajectories from underwater sensor network back to land [4].

Our system differs from these in that our underwater nodes can choose to surface to send large sets of data themselves or to act as a relay for other nodes. This has the advantage of eliminating the acoustic channel bottleneck, but has the drawback of requiring significant energy to surface and surfacing also removes the node from its desired depth for sensing. In this paper, we explore various approaches for choosing surfacing to minimize latency and energy usage.

We also examine greedy approaches for determining the nodes to surface and compare these to optimal methods. The greedy approaches are similar to greedy geographic routing approaches which are near optimal in dense networks; varying approaches exist to improve the performance in more challenging environments $[3,10]$. These approaches focused on homogeneous communication on land-based networks. The performance of these approaches motivates this paper's analysis of the performance of two greedy approaches as applied to our underwater sensor network.

\section{AQUANODE OVERVIEW}

The AquANode system is a flexible underwater sensing and communication system and is shown in Figure 2.

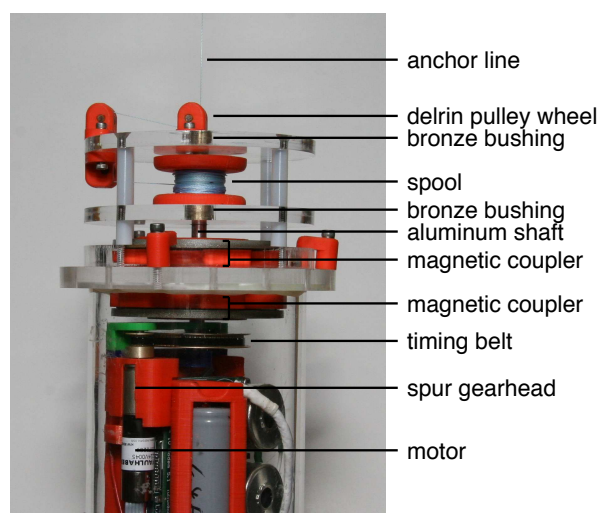

Figure 3: Depth adjustment mechanism details.

We previously described the development of the hardware, electronics, communications systems, and dynamic depth adjustment system for the AQUANODE underwater sensor network system $[6,7,8]$. In the following subsections, we summarize the base AQUANODE system including the depth adjustment attachment and the communication systems.

\subsection{Base System}

The AqUANODE is cylindrically shaped with a diameter of $8.9 \mathrm{~cm}$ and a length of $30.5 \mathrm{~cm}$ with the depth adjustment mechanism. It weighs $1.8 \mathrm{~kg}$ and has a buoyancy of $200 \mathrm{~g}$.

The AQUANODE has three separate onboard processors. At the lowest level, it has a power efficient AtMega164p processor for managing sleep modes and interfacing with low-power sensors. This processor can turn on the highlevel $60 \mathrm{MHz}$, ARM7 LPC2148 processor, which coordinates and controls the overall node and communicates with other nodes. In addition, our acoustic modem has a $600 \mathrm{MHz}$ Blackfin BF533 fixed point DSP processor. All of our nodes have water pressure (for depth) and temperature as well as underwater connectors to allow for a variety of other environmental sensors.

The AquANode has an on-board 60WHr Li-Ion battery. This is sufficient for 2 days of regular acoustic communication, 2 days of continuous motion, 2 weeks of continuous sensing, or up to a year of standby time. The desired deployment time can be achieved by varying the degrees of sensing and communication.

Typically, AQUANODES are moored to an anchor and float in the water mid-column. With the addition of the depth adjustment system, shown in Figure 3 (system inverted for visibility), the AQUANODES are able to dynamically adjust their depth in water of up to $50 \mathrm{~m}$ deep. The depth adjustment system allows the AQUANODES to change depth in water with a speed of $2.4 \mathrm{~m} / \mathrm{min}$ and uses approximately $0.6 \mathrm{~W}$ when in motion.

\subsection{Communication Systems}

In this section we detail the acoustic and radio systems used in the underwater sensor nodes.

Acoustic Modem: The acoustic modem on the AQUANODE platform is a custom design. The hardware and signal processing in the acoustic modem the AQUANODEs use was developed jointly in prior work with Iuliu Vasilescu $[8,11]$. In general, the acoustic modem has a maximum range of 
about 400 meters and typical working range on the order of 100 meters.

At the modem's core is an Analog Devices Blackfin BF533 fixed point DSP processor running at $600 \mathrm{MHz}$. The acoustic modem uses a frequency-shift keying (FSK) modulation with a $30 \mathrm{KHz}$ carrier frequency. The physical layer transmission speed is 300 bits per second.

The MAC layer is a self-synchronizing time division multiple access (TDMA) scheme. Each acoustic modem is given a time slot during which it is the master and controls communication during that slot. The slots are each 4 seconds long and further divided into a master and slave portion. During the master portion of the slot, the master node can transmit a 16 byte packet. A slave, specified by the master during its transmission, can respond during the second half of the slot with a 16 byte packet.

Typically, if there are $N$ acoustic modems, there are $N$ communication slots. By default, node $i$ will own slot $i$. This means that each node is master every $4 N$ seconds. For typical deployments of 10 nodes, each sensor is master every 40 seconds. The acoustic modems also have the ability to give and take slots remotely over the acoustic channel. Thus, a single node may have zero, one or many slots.

Radio Modem: The AQUANode also contains an off-theshelf Aerocomm AC4790 radio [1]. It has a $1 \mathrm{~W}$ transmit power and operates on the $900 \mathrm{MHz}$ band. The claimed range of the radio is $32 \mathrm{~km}$; however, in our experiments, the radio achieved at most $3 \mathrm{~km}$ and typical range was $100 \mathrm{~m}$.

The radio has its own internal packet structure. Nodes can send messages either via broadcast or directed to specific receivers. In the directed mode, the radio will retransmit up to a fixed number of times until it receives an ack for that packet from the receiver. In our system, we typically use broadcast mode so other nodes can snoop in on packet transmissions. In this mode, the radio transmits each packet multiple times (set to 6 in our case). The radio simulates a full-duplex link by using fixed-length time slots for transmission. An individual radio transmits in half of the time slots at most. This reserves time for responses.

\section{COMMUNICATION ENERGY AND TIME ANALYSIS}

The amount of energy used by underwater sensor nodes is critical to the longevity of the system. It is extremely difficult to recharge devices underwater and it is not feasible to regularly replace batteries or devices. In this section, we examine the amount of energy and time used by the two communication systems. We determine the cutoff point for a given network configuration where it is more efficient to surface and use the radio to transmit data back to shore. In our prior work [8], we also investigated a short-range optical communication system that enables an underwater robot to go to individual AQUANODES to download data. In this paper we do not consider the optical communication system since it requires an underwater robot for operation.

\subsection{Acoustic and Radio Energy Analysis}

In this section, we compute the energy usage of the acoustic and radio modem. Since the radio requires surfacing, we also consider the energy required to move the node to the

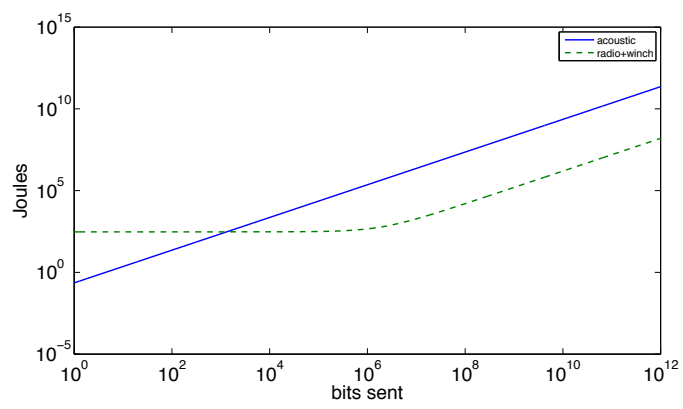

Figure 4: Log-log power per transmission size for message sent $500 \mathrm{~m}$ from a $10 \mathrm{~m}$ depth.

surface. The energy usage numbers are theoretical; however, our computations use realistic numbers that match our empirical energy usage.

The acoustic modem has a maximum transmission power of about 10 watts. However, it typically uses a lower power mode that operates at about 5 watts. Recall that the modem uses a decentralized TDMA algorithm. In each 4 second TDMA slot, we can send and receive a 16 byte packet with 11 bytes of payload. Thus we have a throughput of 5.5 bytes per second. This translates to an acoustic power per bit, $P_{a}$, of:

$$
P_{a}=5 \mathrm{~W} /(5.5 * 8 \mathrm{bits} / \mathrm{sec})=113.6 \mathrm{~mJ} / \mathrm{bit} .
$$

The AquANodes use a 1 watt $900 \mathrm{MHz}$ radio with an $\mathrm{RF}$ baud rate of 76800 bits per second. For broadcast mode, the radio transmits each packet 6 times to ensure reception and, additionally, to simulate full-duplex operation, after each packet transmission, it waits for a time period equal to the packet reception time to allow other radios to transmit. This means the true datarate for this radio is closer to:

$$
76800 / 6 / 2 \mathrm{bits} / \mathrm{sec}=6400 \mathrm{bits} / \mathrm{sec} .
$$

We can then calculate the power per bit using the radio, $P_{r}$, as:

$$
P_{r}=1 \mathrm{~W} / 6400 \mathrm{bits} / \mathrm{sec}=0.16 \mathrm{~mJ} / \mathrm{bit} .
$$

However, to send using the radio, the node must first rise to the surface using the depth adjustment system. The depth adjustment system uses about 0.6 watts and moves at $2.4 \mathrm{~m} / \mathrm{min}$. Thus, we need a power per meter, $P_{w}$ of:

$$
P_{w}=0.6 \mathrm{~W} / 0.04 \mathrm{~m} / \mathrm{sec}=15000 \mathrm{~mJ} / \mathrm{m} .
$$

The total power, $P_{r w}$ to transmit $k$ bits from a depth of $d$ meters using the radio and depth adjustment system (assuming we return to the same location after) is:

$$
P_{r w}=2 d P_{w}+k P_{r}=2 d * 15000 \mathrm{~mJ}+k * 0.16 \mathrm{~mJ} .
$$

\subsection{Energy and Time Comparison}

Figure 4 plots on a log-log scale the total amount of power needed to send various amounts of data based on equations 1 and 5 . In this example, the sensor node wants to transmit the data 500 meters and is at a depth of 10 meters. By plotting log-log, we see the large ranges of power usage depending on message size. As this figure shows, the acoustic system power usage increases at a fast, constant 


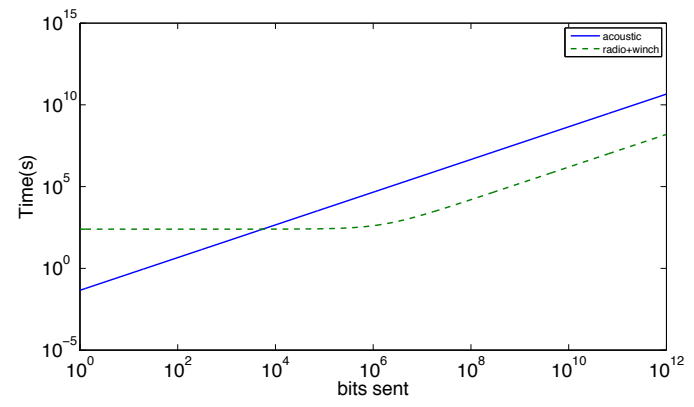

Figure 5: Log-log time to send per transmission size for message sent $500 \mathrm{~m}$ from a $10 \mathrm{~m}$ depth.

rate as each additional bit sent requires the same amount of additional energy. For the radio, the sensor node requires a large initial amount of required energy to bring itself to and from the surface, but then little additional energy per bit to communicate the data after.

The power usage plot shows that there is a clear decision point as to where the node should switch between the communication systems. If sending less than $1328 \mathrm{bits}$, the node should use its acoustic system. To send over 1328 bits, it is more power efficient to first go to the surface with the depth adjustment system, transmit with the radio, and then return to the previous depth.

Figure 5 similarly shows the amount of time it takes to send data. The communication systems have the same ordering as in the power analysis, but the switching point differs as it is faster to send acoustically for less than 5594 bits and faster to send by surfacing and using the radio for more than 5594 bits.

These two plots give two of the possible considerations when trying to decide which communication system is best to use. Nodes determining when to switch between mechanisms can use these equations and knowledge of the network configuration. Once decided, the node then chooses the route upon which it will send the message, for which many solutions exist.

\section{MULTI-MODAL LINK SELECTION ALGORITHMS}

Once a node decides to surface, based on the equations in Section 4, our goal is to determine the best nodes to surface to create a radio path from source to destination. We assume that there are existing underwater communication routes. In this paper, our approach is to determine which of the nodes on the acoustic path should surface to create the radio route. The naive approach would be to have all nodes surface, but this is inefficient since the radio communication range is typically larger than the acoustic range. Selecting the best set of nodes for a successful radio path may depend partially on the goals; for example, we may want to minimize the number of hops in the radio route or we may want to conserve energy by minimizing the total distance the nodes travel.

To achieve these goals, we explore and compare localgreedy approaches versus the global optimal algorithms. The first approach is a greedy approach where the furthest node within expected radio range of the current node sur- faces (Greedy Furthest Radio). While easy to implement, the approach may need to conservatively estimate radio range to ensure connectivity. We can compare this to a global shortest path algorithm that selects the set of nodes that minimizes hops, providing a Min-Hop Furthest Radio approach.

A drawback of the Furthest Radio approaches is that they do not consider depth, which is the main power use of any approach. We therefore can modify the approach to instead select the node closest to the surface of all neighbors within radio range (Greedy Shallowest Radio). This will reduce the distance traveled by the nodes, but may not select a minimal nor optimal set of nodes. For instance, consider a network with nodes with continuously increasing depth. In this case, the Greedy Shallowest Radio algorithm will cause all nodes to surface. To select an optimal set of nodes, we compute the Min-Hop Shallowest Radio path using the current node depth to create weights. This ensures that the overall travel distance is minimized.

\section{SIMULATION RESULTS}

We perform an initial analysis of our set of algorithms as well as worst-case approach. The worst case is having every node rise on the path, which we call All Surface. To compare all these algorithms, we implemented a simulator in Matlab. This simulator uses the object-oriented package to instantiate independent nodes with transmit and receive queues, randomized topologies including depths, and models of each communication method.

The results are from a set of simulations with nodes in a line topology for network with sizes ranging from 10 to 100 nodes. Each node is randomly placed in the line at a depth between $0 \mathrm{~m}$ and $20 \mathrm{~m}$ and a location within $65 \mathrm{~m}$ of its neighbors (a safe maximum range of the acoustic radio). Due to the randomization, we compute 50 iterations to find the mean behavior of each of our approaches. We do not consider packet loss in this simulation, which is a critical factor, since we are interested in comparing these different approaches under optimal conditions. In future work we will analyze the impact of packet loss on these approaches.

Figure 6(a) shows an example configuration with 10 nodes and the four main algorithms we explore. Nodes are slightly offset from each other for visibility. Note that each approach causes a different set of nodes to surface. For these algorithms, we examine two key parameters. The first is the overall distance traveled by the nodes in the network, since this dominates the energy usage for the radio. The second is the delay between when the source decides to surface to when the destination node rises and completes the link.

Figure 6(b) shows the average distance traveled by nodes (including those that do not move). The difference in the energy usage between different approaches largely depends on this distance. As expected, the worst-case of all nodes rising has a significantly higher cost. The approaches aiming to minimize hops, Greedy Furthest Radio and Min-Hop Furthest Radio, perform similarly and not as well as those that try to minimize motion. At times the Greedy Furthest Radio actually outperforms the Min-Hop Furthest Radio approach, which is reasonable since neither consider depth. Interestingly, while the worst-case scenario for Greedy Shallowest Radio is the same as All Surface (in the case where 

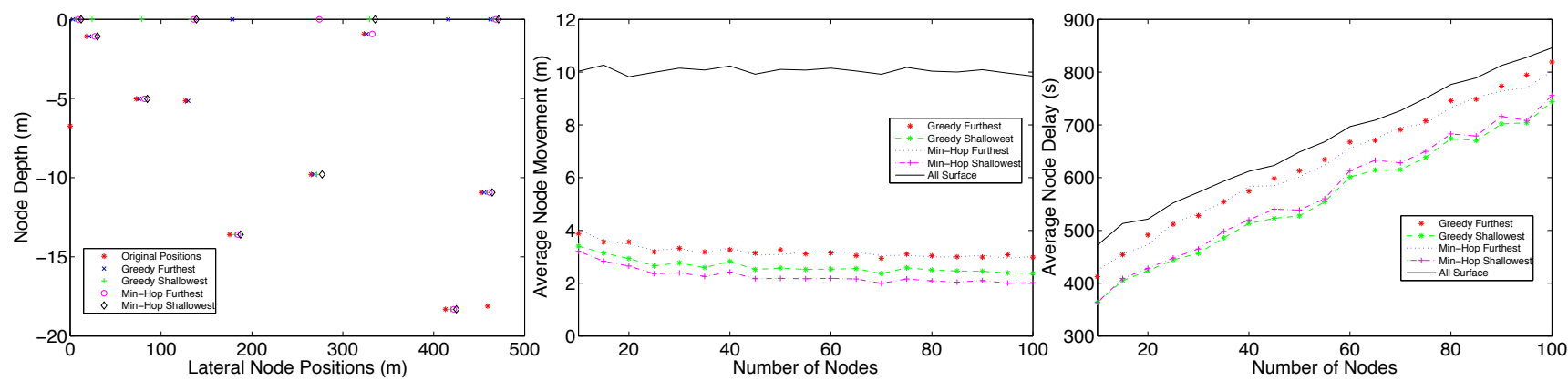

Figure 6: For each algorithm, (a) Example start and end configurations, (b) Average distance traveled per node, and (c) Average delay between source node's decision to send a message and the complete radio path.

depths are monotonically increasing), the average performance in these randomized trials are very close to the optimal Min-Hop Shallowest Radio. The greedy approach has the advantage of requiring virtually no communication overhead in determining the route, which is critical in bandwidth and energy constrained underwater networks. This also suggests that for more generic networks with multiple source and sinks of data greedy approaches will also work well.

Figure 6(c) shows the delay from a node's decision to rise to full radio path creation. This data is optimistic in that it assumes perfect acoustic communication such that a node will know to rise on the first relayed acoustic message it receives from the source node. The total delay to create the link consists of the total time to send the acoustic rise message to all nodes plus the total time for the selected nodes to rise. As seen in this figure, the 400 seconds needed for the deepest node along the path to surface (on average at 16 meters deep) initially dominates the delay time. However, as the network size grows, the time needed to relay messages, optimistically 4 seconds per hop, starts to dominate the link creation time. This suggests a focus on minimizing the number of communication steps in the algorithm, especially as network sizes grow, which strongly supports using greedy approaches with only local information.

\section{CONCLUSIONS AND FUTURE WORK}

To reduce communication costs and increase network lifetimes, we utilize an underwater sensor network with both acoustic and surface radio communication mechanisms. We analyze these mechanisms and develop equations to enable nodes to choose between them based on energy and time. We develop a set of algorithms to determine the set of nodes that should surface on a given route once message sizes increase beyond reasonable acoustic packets. Our preliminary analysis shows that the best method considers a greedy approach where nodes closest to the surface rise. In moving forward, we plan to expand this approach to handle threedimensional networks and multi-packet path optimization. We also plan to examine the impact of both acoustic and radio packet loss on the system and we will ultimately implement this on our AQUANODE platform.

\section{ACKNOWLEDGMENTS}

We are grateful to NSF RI (IIS-1116221) and NSF CNS (CSR-1217400 and CSR-1217428), which partially supported this work.

\section{REFERENCES}

[1] Laird technologies - the world leader in bluetooth, 802.11, ZigBee and proprietary RF modules. http://www.lairdtech.com/wireless/.

[2] I. F. Akyildiz, D. Pompili, and T. Melodia. State-of-the-art in protocol research for underwater acoustic sensor networks. In Proceedings of the 1st ACM international workshop on Underwater networks, WUWNet '06, pages 7-16, New York, NY, USA, 2006. ACM.

[3] N. Arad and Y. Shavitt. Minimizing recovery state in geographic ad-hoc routing. In Proceedings of the 7th ACM international symposium on Mobile ad hoc networking and computing, MobiHoc '06, pages 13-24, New York, NY, USA, 2006. ACM.

[4] B. Chen, P. C. Hickey, and D. Pompili. Trajectory-aware communication solution for underwater gliders using WHOI micro-modems. In Sensor Mesh and Ad Hoc Communications and Networks (SECON), 2010 7th Annual IEEE Communications Society Conference on, pages 1-9, 2010.

[5] J. Cui, J. Kong, M. Gerla, and S. Zhou. The challenges of building mobile underwater wireless networks for aquatic applications. Network, IEEE, 20(3):12-18, June 2006.

[6] C. Detweiler, M. Doniec, I. Vasilescu, E. Basha, and D. Rus. Autonomous depth adjustment for underwater sensor networks. In Proc. of the Intl Workshop on UnderWater Networks (WUWNet), pages 12:1-12:4, 2010.

[7] C. Detweiler, M. Doniec, I. Vasilescu, and D. Rus. Autonomous depth adjustment for underwater sensor networks: Design and applications. IEEE Transactions on Mechatronics, 17(1):16-24, 2012.

[8] C. Detweiler, I. Vasilescu, and D. Rus. An underwater sensor network with dual communications, sensing, and mobility. In OCEANS 2007 - Europe, pages 1-6, 2007.

[9] J. Rice. SeaWeb acoustic communication and navigation networks. In Proceedings of the International Conference on Underwater Acoustic Measurements: Technologies and Results, 2005.

[10] G. Tan, M. Bertier, and A. Kermarrec. Visibility-Graph-Based Shortest-Path geographic routing in sensor networks. In INFOCOM 2009, IEEE, pages 1719-1727, Apr. 2009.

[11] I. Vasilescu. Using Light Underwater: Devices, Algorithms and Systems for Maritime Persistent Surveillance. PhD thesis, MIT, 2009.

[12] Z. Zhou, H. Yan, S. Ibrahim, J. Cui, Z. Shi, and R. Ammar. Enhancing underwater acoustic sensor networks using surface radios: Issues, challenges and solutions. In G. Ferrari, editor, Sensor Networks, Signals and Communication Technology, pages 283-307. Springer Berlin Heidelberg, 2009. 